\title{
Microstructured Silicone Substrate for Printable and Stretchable Metallic Films
}

\author{
Adam P. Robinson, ${ }^{*}$ Ivan Minev, Ingrid M. Graz, and Stéphanie P. Lacour \\ Nanoscience Centre, Department of Engineering, University of Cambridge, $11 \mathrm{~J}$. J. Thomson Avenue, Cambridge CB3 0FF, \\ United Kingdom
}

Supporting Information

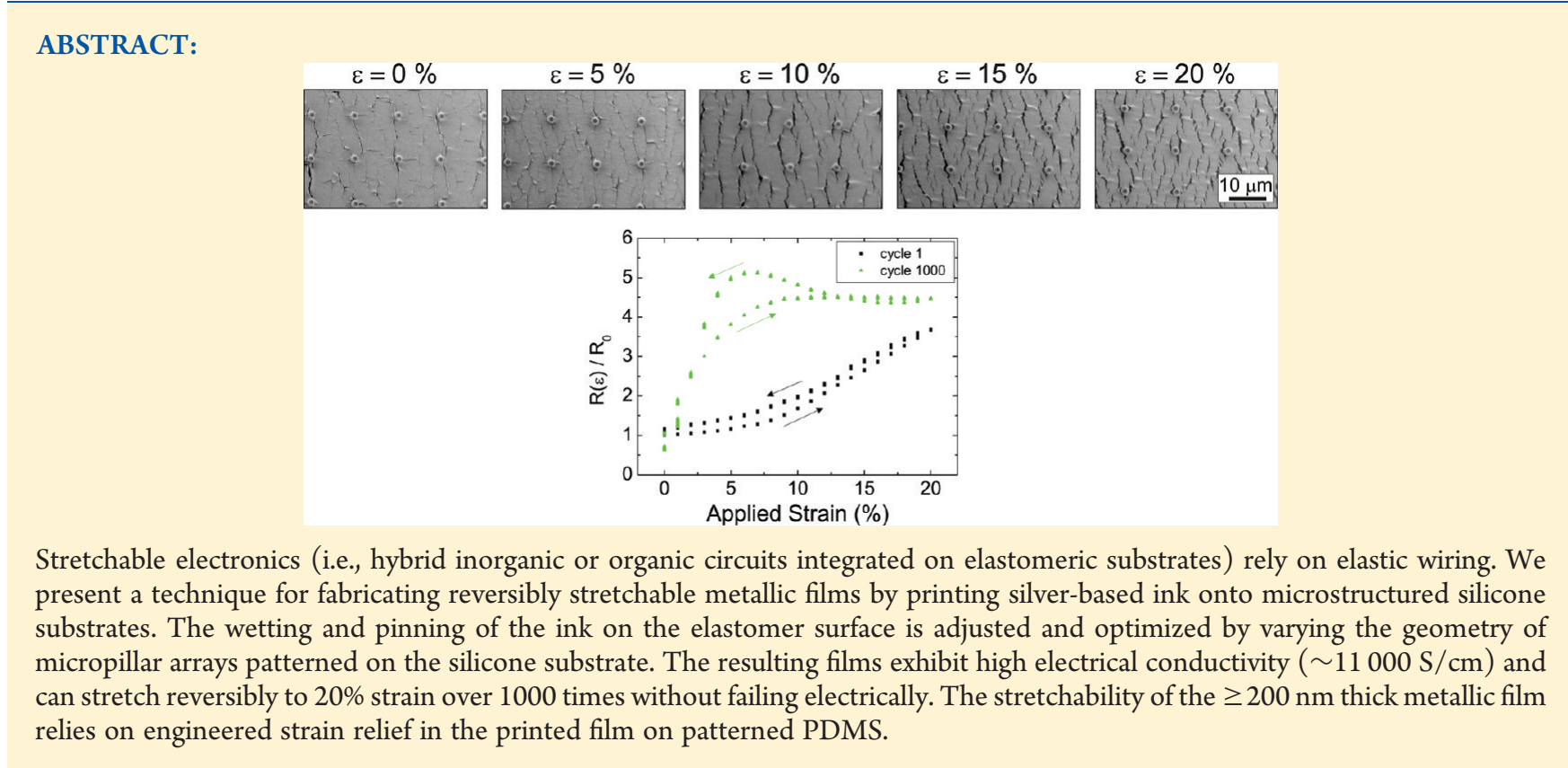

\section{INTRODUCTION}

Silicone elastomers have been recently implemented as a new class of substrates for microelectronics and transducing circuits. ${ }^{1-5}$ The mechanical compliance of silicones promises applications where the electronic circuitry is no longer constrained to the $2 \mathrm{D}$ plane but rather can conform to the human body ${ }^{6,7}$ and allow for consumer electronics products with changeable form factors. ${ }^{8}$ The electro-mechanical integrity of the stretchable circuits relies on elastic wiring (i.e., electrical conductors) that can reversibly sustain mechanical loading and ensure reliable electrical conduction between the electrical devices. Ideally, a stretchable conductor should have a high electrical conductivity, be easily patterned onto the elastomer surface, and be robust to large mechanical stretch. Elastic wiring can be prepared by patterning thin or thick metal films on the silicone membrane $e^{1,4,5,9,10}$ or by preparing conducting composite silicones. ${ }^{3}$ The former have high electrical conductivity $\left(>10^{6} \mathrm{~S} / \mathrm{m}\right)$ but require complex patterning processes; the latter are extremely robust to mechanical stretching but have low conductivity $(\sim 1000 \mathrm{~S} / \mathrm{m})$ and are not readily compatible with microfabrication techniques. Inkjet printing or plotting offers a simple patterning process to obtain high-resolution complex metallic patterns. They are rapid and additive patterning techniques that do not need multiple fabrication steps and sacrificial photoresists as required in evaporation or electrodeposition combined with photolithography. The printing of metallic inks onto silicone surfaces appears to be a promising alternative to write stretchable, low-resistance conductors directly.

The PDMS (poly(dimethysiloxane)) surface is very hydrophobic and has a lower surface energy than most organic polymers $(<30 \mathrm{mN} / \mathrm{m})$. Exposure to oxygen or corona plasma alters the PDMS surface, both chemically and physically. Cracking of the exposed surface and increased wettability are usual observations, even after short (tens of seconds) plasma exposure. When stored in air, the exposed PDMS surface gradually recovers its initial hydrophobicity (within minutes to hour of exposure). Printing liquid ink on the PDMS surface therefore requires control of the elastomer surface properties. In this letter, we demonstrate that silver lines can be reliably plotted from soluble

\section{Received: August 12, 2010}

Revised: $\quad$ March 7, 2011 
silver cluster ink onto microstructured PDMS surfaces. We report that structuring the PDMS surface with arrays of micrometer-scale pillars enables reliable wetting and pinning of the ink so that metallic stripes of controlled geometry can be printed over large-area surfaces. Moreover, the printed conductors can reversibly stretch by tens of percent strain. The micropillar arrays act as in situ strain relief and prevent crack propagation across the metallic film.

\section{EXPERIMENTAL SECTION}

Inverted micropillar molds are produced on silicon wafers using a photoresist (AZ5214e, MicroChemicals) patterned by photolithography. The silicon wafers are first baked for $2 \mathrm{~h}$ at $200{ }^{\circ} \mathrm{C}$ to remove moisture and coated with hexamethyldisilazane (HMDS) spun for $10 \mathrm{~s}$ at $2000 \mathrm{rpm}$ to produce an adhesion layer. The photoresist is spin coated at $2000 \mathrm{rpm}$ for $30 \mathrm{~s}$ and prebaked for $2 \mathrm{~min}$ at $110^{\circ} \mathrm{C}$ to obtain a $2-\mu \mathrm{m}$ thick layer. UV exposure is carried out for $4 \mathrm{~s}$ using a Suss MJB3 mask aligner through a chromium mask with the relevant pattern. The insulated pattern is developed for $30 \mathrm{~s}$ in a 1:4 developer (MIF351B, MicroChemicals) to deionized (DI) water mixture and finally rinsed in DI water. Before PDMS casting, the molds are perfluorinated to reduce adhesion between the PDMS and resist layer. Microstructured PDMS is produced by casting Dow Corning Sylgard 184 mixed in a ratio of 10:1 (polymer: cross-linker ratio) against the molds for a period of $24 \mathrm{~h}$ at $65{ }^{\circ} \mathrm{C}$. Smooth PDMS surfaces are prepared by casting against continuous photoresist films on silicon wafers using the same conditions minus the resist exposure and development steps. Printing is carried out using a SonoPlot GIX Desktop Microplotter at a speed of $2 \mathrm{~mm} / \mathrm{s}$ using a glass vial with a tip that produces a printed line of $220 \mu \mathrm{m}$. Single 15-mm-long lines are printed onto the substrates using the SonoPlot software. Prints are activated by heating on a hot plate at $150^{\circ} \mathrm{C}$ in air for $10 \mathrm{~min}$. Contact angle measurements are carried out using a goniometer. Static contact angles are measured after placing a $5 \mu \mathrm{L}$ drop of ink on the PDMS surface. Dynamic contact measurements are made using a syringe attached to a stepper motor, which increased (advancing) and decreased (receding) the drop volume at a rate of $1 \mu \mathrm{m} / \mathrm{s}$ from an initial drop size of $5 \mu \mathrm{m}$ up to $15 \mu \mathrm{m}$ and back. The print morphology was studied using an optical microscope. Print thicknesses were studied in cross section using an SEM. Printed conductors were mechanically cycled to a maximum applied strain of $20 \%$ using a computer-controlled, motorized uniaxial stretcher. Compliant electrical contacts are prepared using silver wire, conductive silver paste, and PDMS pads. Electrical resistance data with cyclic strain was recorded using a Keithley 2410 every 500 ms while increasing the strain at a rate of $0.15 \mathrm{~mm} / \mathrm{s}$ in $1 \%$ steps, with a 60 s pause between steps. Extended strain cycles were performed at a faster rate of $1.5 \mathrm{~mm} / \mathrm{s}$ while measuring the resistance only at maximum and minimum strains. The surface topography of printed lines was studied during stretch cycles using an FEI/Philips XL30 E-SEM-FEG containing a computer-controlled, motorized, uniaxial stretcher. Images were analyzed using Image J software.

\section{RESULTS AND DISCUSSION}

PDMS has a low surface energy $\left(19.8 \mathrm{mN} / \mathrm{m}\right.$ at $20{ }^{\circ} \mathrm{C}$ for Sylgard 184, Dow Corning) and thus shows lyophobic surface properties. The measured contact angle of a $5 \mu \mathrm{L}$ drop of water with an as-prepared (untreated) PDMS surface is $116 \pm 1^{\circ}$. The large coefficient of thermal expansion of silicone, $>200 \mathrm{ppm} /{ }^{\circ} \mathrm{C}$, compared to that of metals, $<20 \mathrm{ppm} /{ }^{\circ} \mathrm{C}$, guides the selection of metallic inks with a low sintering or activation temperature. The reliable printing of conductive ink on the PDMS surface therefore requires control of the elastomer surface properties and a careful selection of the metallic ink. In the proposed printing on PDMS approach, the elastomer surface is first patterned into an array of pillarlike microstructures; the rough yet controlled surface allows for the control of ink wetting and pinning. The ink is plotted onto the elastomer surface by continuously forcing the ink drop at the opening of the resonating glass nozzle onto the surface rather than jetting from the nozzle. Low-activationtemperature ink is selected to form the metallic film: InkTecIJ-series transparent, conductive silver-based (Ag) ink consists of a complex containing silver atoms and organic ligands, which are destroyed during activation by heating at $130-150{ }^{\circ} \mathrm{C}$ in air for 10 minutes. The ink is thus activated at a significantly lower temperature than the majority of conducting Ag inks, which require sintering of metallic nanoparticles at temperature in the 250 to $350{ }^{\circ} \mathrm{C}$ range. ${ }^{11}$ Being solvent-based, the surface tension of InkTec ink is low $\left(30 \mathrm{mN} / \mathrm{m}\right.$ at $\left.25^{\circ} \mathrm{C}^{12}\right)$; the contact angle of a $5 \mu \mathrm{L}$ drop of ink on a smooth PDMS surface is measured to be $50 \pm 1^{\circ}$, which is well within the lyophilic range. Despite this, printed lines of InkTec ink onto a smooth PDMS surface result in discontinuous features. Figure la shows the surface of a $200-\mu \mathrm{m}$ wide line printed onto smooth PDMS, after curing, indicating the problems associated with ink dewetting. Note that dewetting occurs during ink drying before curing. Liquid dewetting on the PDMS surface may be reduced by increasing the substrate's surface energy using oxygen/ozone plasma treatment. However, even short (tens of seconds) treatments produce very thin $(<10 \mathrm{~nm})$ yet brittle silicon oxide $\left(\mathrm{SiO}_{x}\right)$ films at the PDMS surface; ${ }^{13}$ subsequent metal film deposition and mechanical stretch lead to extensive film wrinkling and cracking, thereby seriously limiting the structure's elasticity and electrical integrity. Altering the roughness and topography of the PDMS surface is an alternative to controlling its wettability. ${ }^{14-16}$

A model of fluid invasion based on thermodynamic arguments was proposed by Bico et al. ${ }^{16}$ and corroborated by experimental
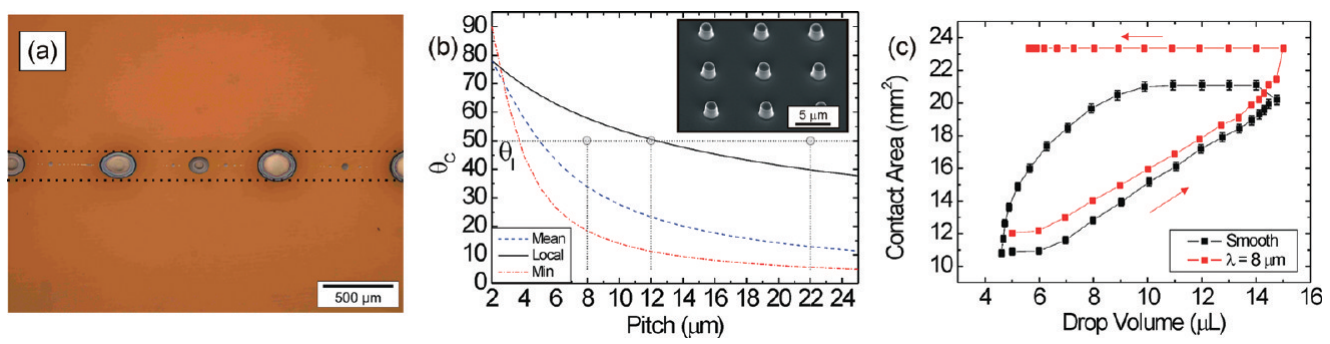

Figure 1. (a) Discontinuous printed silver conductor on smooth PDMS. (b) $\theta_{\mathrm{C}_{\text {menan }},} \theta_{\mathrm{C}_{\text {(ow }}}$ and $\theta_{\text {min }}$ as a function of pitch $\lambda$ for 2- $\mu \mathrm{m}$-diameter, $2-\mu \mathrm{m}$ high pillars; the horizontal dotted line indicates $\theta_{\mathrm{I}}$ for smooth PDMS/InkTec ink, and the vertical dotted lines show the values of $\lambda$ selected. The inset shows an SEM image of a PDMS pillar array with $\lambda=8 \mu \mathrm{m}$. (c) Dynamic contact area data for smooth and patterned $\lambda=8 \mu \mathrm{m}$ PDMS substrates. 
data on an idealized rough surface. On an ideally smooth surface in air, ignoring the effects of gravity, a drop of liquid will form an intrinsic static contact angle, $\theta_{\mathrm{I}}$, dependent on the surface energy of the solid and the surface tension of the liquid. On a rough surface of the same material, the apparent static contact angle, $\theta_{\mathrm{A}}$ will also depend on the geometrical parameters of the surface pattern. ${ }^{17-19}$ Using an idealized structured surface patterned with micrometer-sized pillars arranged in square arrays, Bico et al. calculated a critical angle, $\theta_{\mathrm{C}}{ }^{16}$ as

$$
\theta_{\mathrm{C}}=\arccos \left(\frac{1-\phi_{\mathrm{S}}}{r-\phi_{\mathrm{S}}}\right)
$$

where $r$ is the roughness of the solid surface, calculated as the ratio of the actual surface area over its projected one (and $>1$ ) and $\varphi_{\mathrm{S}}$ is the solid surface fraction remaining dry (if $0<\theta_{\mathrm{I}}<90^{\circ}$, this represents the pillar tops). If $\theta_{\mathrm{I}}<\theta_{\mathrm{c}}$, the micropattern will aid liquid invasion and hinder dewetting; if $\theta_{\mathrm{I}}>\theta_{\mathrm{C}}$, the roughness will hinder liquid invasion and not hinder dewetting. For the general case (disordered surfaces), a mean value of $\theta_{\mathrm{C}}\left(\theta_{\mathrm{C}_{\text {mean }}}\right)$ may be calculated for the whole surface. However, for the discontinuously textured surfaces studied by Bico and by us, with pillar rows separated by regions of smooth surface providing activation barriers for the liquid contact line to jump, care must be taken when applying the theory to the separate cases of fluid invading a dry surface and fluid dewetting an invaded surface. ${ }^{16}$ For the case of fluid invasion of a dry surface, for a contact line pinned on a row of pillars to jump to the next row it must be able to touch it (i.e., the menisci around each pillar must be large enough to reach the next row of pillars). This may be approximated as a $1 \mathrm{D}$ prism, ${ }^{16}$ where the critical angle to ensure that the liquid front jumps rows is given by

$$
\tan \theta_{\min }=\frac{\delta}{D}
$$

where $\delta$ is the pillar height and $D$ is the distance between pillars. Fluid will not invade the surface if $\theta_{\mathrm{I}}>\theta_{\text {min }}$. For the case of dewetting, where the surface is already coated with a liquid film, the liquid front must dewet individual pillar rows: for isolated pillar rows, both $r$ and $\varphi_{\mathrm{S}}$ are locally significantly higher than the mean value, producing a higher value for $\theta_{\mathrm{C}_{\text {local }}}$ than $\theta_{\mathrm{C}_{\text {mean }}}$. If $\theta_{\mathrm{I}}<$ $\theta_{\mathrm{C}_{\text {local }}}$ the pillar rows will hinder dewetting; if $\theta_{\mathrm{I}}>\theta_{\mathrm{C}_{\text {local }}}$ they will not. Note that $\theta_{\mathrm{C}_{\text {local }}}$ is calculated in the same way as $\theta_{\mathrm{C}_{\text {mean }}}$, using eq 1 , except for the locally higher values for $r$ and $\varphi_{S}$ for an individual pillar row. If fluid satisfying the case $\theta_{\min }<\theta_{\mathrm{I}}<\theta_{\mathrm{C}_{\text {local }}}$ is deposited on the surface, it will pin on the pillar rows, neither invading nor dewetting them. ${ }^{16}$ It is therefore possible to control how fluid invades the material surface and how it dewets an invaded surface by adjusting the pillar geometry independently of the chemistry. For a given pillar height $\delta$ and diameter $D$, we alter the pitch $\lambda$ in order to control $\theta_{\mathrm{C}_{\text {local }}}$ and $\theta_{\text {min }}$. Several groups have demonstrated that the capillary forces felt by a liquid on such a surface can be used to control the dewetting of liquid polymers to produce accurate mold reproduction in a process labeled capillary force lithography. ${ }^{20,21}$ Here we demonstrate that the idealized roughness of pillar arrays can also be used to produce very uniform printed film conductors on PDMS.

Casting PDMS against a photoresist mold produces surfaces with pillar arrays. The pillars' height and diameter are set to $2 \mu \mathrm{m}$, the smallest reproducible diameter over $>1 \mathrm{~cm}^{2}$ surface area that our UV lithography allows. Pillars with an aspect ratio larger than 1 often collapse upon release from the mold and therefore are avoided. Figure $1 \mathrm{~b}$ presents the predicted critical contact angles for $2-\mu \mathrm{m}$-tall, $2-\mu \mathrm{m}$-diameter pillars calculated for both the mean and the local roughness, as a function of pitch $\lambda$. Using the contact angle of Intek ink on smooth PDMS, $\theta_{\mathrm{I}}=50 \pm 1^{\circ}$, three values of pitch are selected that satisfying three states: $\theta_{\mathrm{I}}<\theta_{\mathrm{C}_{\text {local }}}$ (hindering dewetting, $\lambda=8 \mu \mathrm{m}, \theta_{\mathrm{C}_{\text {local }}}=58.2^{\circ}$ ), $\theta_{\mathrm{I}} \approx \theta_{\mathrm{C}_{\text {local }}}$ $\left(\lambda=12 \mu \mathrm{m}, \theta_{\mathrm{C}_{\text {local }}}=50.7^{\circ}\right)$, and $\theta_{\mathrm{I}}>\theta_{\mathrm{C}_{\text {local }}}$ (not hindering dewetting, $\left.\lambda=22 \mu \mathrm{m}, \theta_{\mathrm{C}_{\text {local }}}=39.9^{\circ}\right)$. All three states also satisfy the condition $\theta_{\mathrm{I}} \gg \theta_{\min }$ ensuring that liquid will not invade the dry surface, which is important to maintain control of the printed ink: if invasion occurs, control of the print dimensions will be lost. The corresponding three types of surface patterns are prepared.

The inset to Figure $1 \mathrm{~b}$ shows a scanning electron microscope (SEM) image of an array of pillars with $\lambda=8 \mu \mathrm{m}$, illustrating highly uniform pillar production. The slight taper toward the tip is due to the positive sidewall slope of the photoresist mold. To indicate the effect of the pillar structures on the dewetting of ink from the surface, dynamic contact angle measurements are carried out on both smooth and patterned PDMS substrates. Figure 1c shows the contact area as a function of ink drop volume during advancing and receding for a single cycle on a smooth substrate and on a pillar substrate with $\lambda=8 \mu \mathrm{m}$. (Similar results are observed for all patterned substrates, but for clarity only one example is shown.) The contact area on the smooth surface increases during the advancing stage and, with some hysteresis, recedes during the receding stage. Note that the contact area recedes to its initial area after a single cycle, indicating that ink is not well pinned to the surface. The contact area on the microstructured surface increases in a manner similar to that of the smooth sample during the advancing stage. However, during the receding stage, as the drop volume is reduced, the contact area remains constant and at its maximum $\left(S=23 \mathrm{~mm}^{2}\right)$. This indicates that the ink pins to the patterned surface because of capillary wicking forces between the pillars. Interestingly, similar pinning mechanisms and the maximal contact area are observed across surfaces with different $\lambda$ values. However, it is worth noting that the dewetting force applied during the receding stage of the dynamic contact angle measurements does not accurately simulate the final stages of drying during ink activation.

Figure 2 shows optical microscope images of three printed conductors on microstructured substrates with all values of $\lambda$ after curing. All conductors are printed in one pass. The resulting prints are extremely uniform and continuous, showing that the ink is well pinned to the substrate. Highly uniform prints can be obtained over a length of several centimeters. The initial printed line width is $220 \mu \mathrm{m}$; the final (cured) line width decreases with increasing $\lambda$. For $\lambda=22 \mu \mathrm{m}$, where $\theta_{\mathrm{I}}>\theta_{\mathrm{C}_{\text {local }} \text {, significant }}$ dewetting is observed and residual ink is clearly visible at the base of pillars in the dewetting region, initially covered during printing. This phenomenon has also been observed for dewetting fluids around isolated pinning sites. ${ }^{22}$ For $\lambda=12 \mu \mathrm{m}$, where $\theta_{\mathrm{I}} \approx$ $\theta_{\mathrm{C}_{\text {local }}}$ and the final line width is slightly lower than the printed width, no evidence of dewetting is observed on the neighboring pillars. It should be noted that the reduction in line width is approximately equal to the pillar pitch. The edge of the printed line must fall somewhere between two rows of pillars; the liquid front recedes until it reaches the next pillar row. Because this occurs on both sides of the print, a reduction in line width of between 0 and $2 \lambda$ can be expected. For $\lambda=8 \mu \mathrm{m}$, where $\theta_{\mathrm{I}}<$ $\theta_{\mathrm{C}_{\text {local }}}$ no dewetting is observed, indicating that the ink is strongly pinned to the surface. Because the pillar pitch is lower, the observed reduction in line width is also lower. As the printed line 

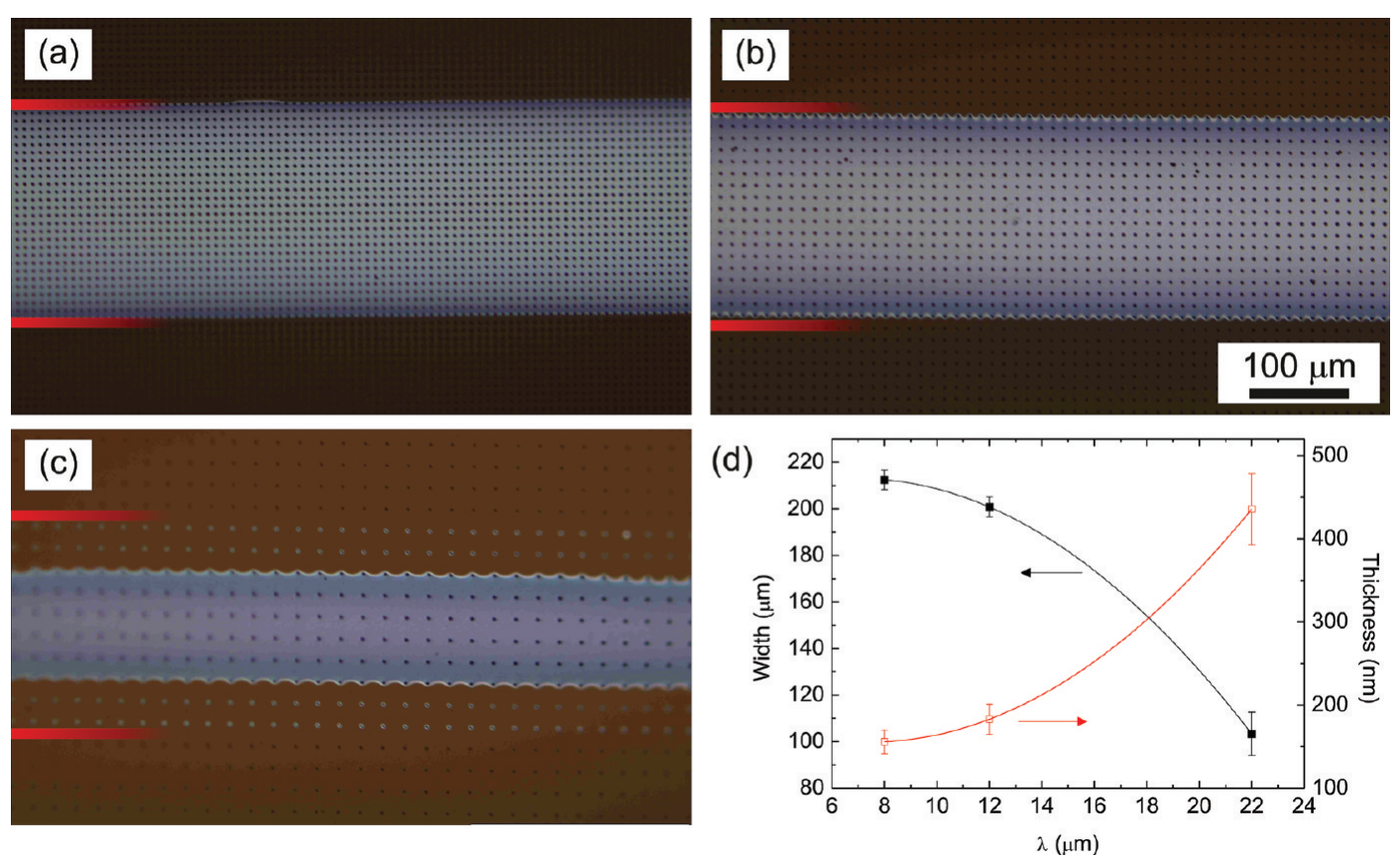

Figure 2. Optical microscope images of the surface of printed silver lines on patterned PDMS after curing $\left(150^{\circ} \mathrm{C}, 10 \mathrm{~min}\right)$ for $\lambda=8(\mathrm{a}), 12(\mathrm{~b})$, and $22 \mu \mathrm{m}$ (c). The print direction is from left to right, and the red lines indicate the initial printed line width before curing. (d) Plot showing line width and thickness as a function of $\lambda$.

edge recedes to the nearest pillar row, print resolution is limited by pillar pitch. At constant ink volume, the width of the printed line decreases because of dewetting and its thickness increases. Figure $2 \mathrm{~d}$ plots the recorded lines' width and thickness as a function of $\lambda$. The resulting conductors' thickness ranges from just under $200 \mathrm{~nm}$ to over $400 \mathrm{~nm}$. Note that the conductor thickness is significantly less than the pillar height, producing arrays of holes in the final printed conductor. dc electrical measurements carried out on unstretched but cured printed lines give high conductivity for all samples of $11000 \pm 3000 \mathrm{~S} / \mathrm{cm}$. The film on PDMS is slightly more resistive than a film with the same geometry printed on a smooth polyimide substrate and cured under the same conditions $(27000 \pm 6000 \mathrm{~S} / \mathrm{cm})$.

To demonstrate the stretchability of printed silver conductors on patterned PDMS, the systematic recording of their electrical resistance is monitored as a function of applied uniaxial (1D) strain. The conductor is $5 \mathrm{~mm}$ long; the PDMS substrate is clamped between two mechanical jaws away from the conducting stripe and strained from 0 to $20 \%$ and back in $1 \%$ steps with a $60 \mathrm{~s}$ pause at each strain value. Figure 3 a shows the normalized change in printed conductor resistance $R(\varepsilon) / R_{0}$ as a function of applied strain for each of the prints shown Figure 2, where $\varepsilon$ is the applied strain and $R_{0}$ is the unstrained resistance. It is interesting to note that all of the conductors stretch to up to $20 \%$ strain without a loss of conductivity, whereas evaporated gold films thicker than $100 \mathrm{~nm}$ fail electrically at strains of a few percent. ${ }^{23}$ The densest pillar array $(\lambda=8 \mu \mathrm{m})$ results in the largest change in resistance, $R_{\max } \approx 7 R_{0}$, with little hysteresis. The least-dense array, which results in the thickest print, produces a lower change in resistance, $R_{\max } \approx 3 R_{0}$, but a large amount of hysteresis, with significant relaxation observed while resting at each percent strain. The best results are obtained for the intermediate density $(\lambda=12 \mu \mathrm{m})$ with a small resistance change and little hysteresis. This substrate has a value of $\lambda$ that is just low enough to prevent
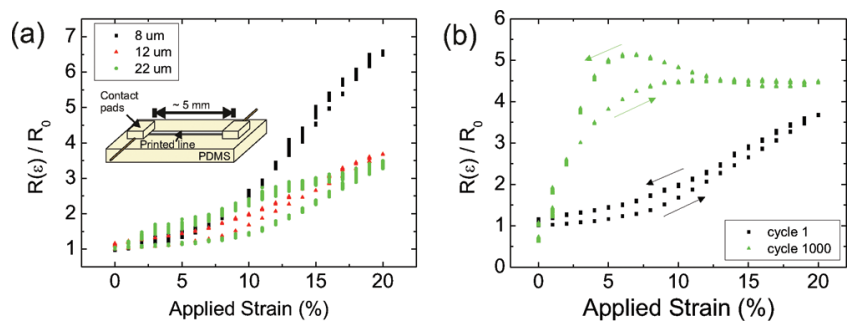

Figure 3. (a) dc resistance measured as a function of applied strain for printed InkTec films: cycle 1 for all values of $\lambda$, where the inset shows a schematic diagram of the measurement setup. Values of $R_{0}$ for all lines are measured to be $140 \pm 20 \Omega$. (b) Cycles 1 and 1000 for the $\lambda=12 \mu \mathrm{m}$ substrate.

the dewetting of ink during curing, avoiding the production of a thick print (Figure 2d). Figure $3 \mathrm{~b}$ shows dc resistance data for the $\lambda=12 \mu \mathrm{m}$ sample under extended cycling for the 1st and 1000th stretch cycles to $20 \%$ maximum strain. During cycle 1 , the resistance increases (and decreases) with the applied strain. At maximum strain, the resistance $R(\varepsilon)$ is found to be $\sim 3 R_{0}$. This resistance increase is comparable to that observed in stretchable thin gold films (50 nm thick) on smooth PDMS. ${ }^{24}$ The $R(\varepsilon)$ response is nonlinear, with a steep increase (decrease) for strain above $10 \%$. After 1000 stretch cycles, the conductor's resistance at maximum strain is about $4 R_{0}$ and the $R(\varepsilon)$ profile shows a plateau for strains larger than $5 \%$. The evolution of the $R(\varepsilon)$ profile from a pseudolinear response to a "saturating" one upon repeated stretching is similar to that reported for thin gold films on PDMS. ${ }^{24}$

Two-micrometer-diameter pillars on the substrate surface with height greater than the print thickness lead to the inclusion of similarly sized circular holes in the printed conductor. This type of feature has been studied numerically by Tucker et al. as a 


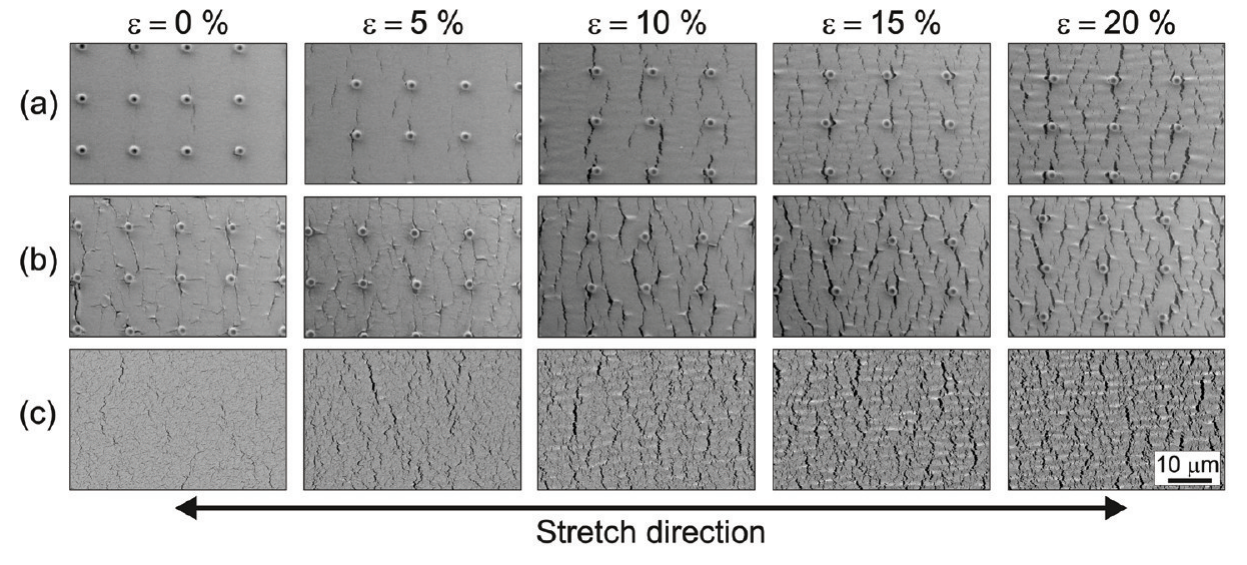

(d)

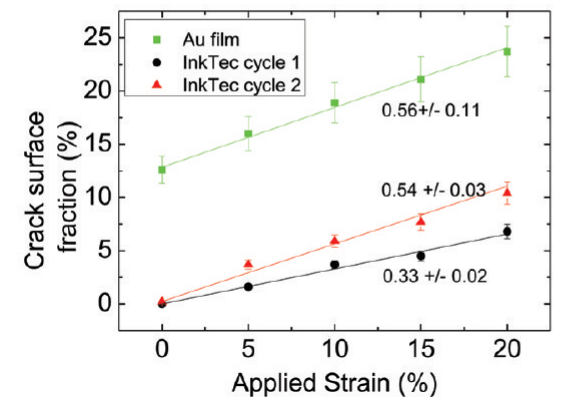

(e)

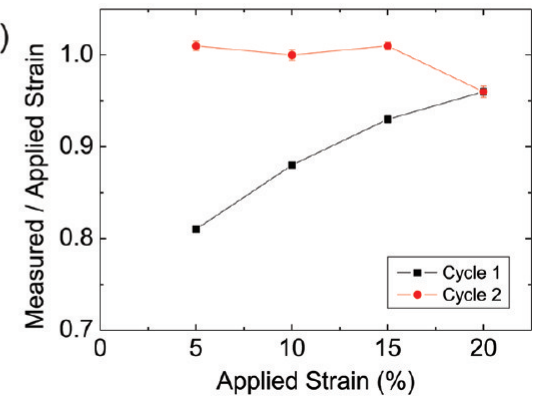

Figure 4. ESEM images of conductor surfaces while under applied uniaxial strain $(\varepsilon=0,5,10,15$, and 20\%). The scale bar is $10 \mu \mathrm{m}$. InkTec ink print on patterned PDMS during (a) cycle 1 and (b) cycle 1000. (c) Fifty-nanometer-thick evaporated gold on smooth PDMS during cycle 1 for comparison. (d) Average cracks in the surface over the conductor surface area as a function of applied strain for gold and InkTec films. (e) Ratio of measured strain to applied strain in a printed film on patterned PDMS as a function of applied strain for the first and second stretch cycles.

mechanism of strain relief in thin metallic films. ${ }^{25}$ It was demonstrated that if the film can stretch only in-plane then strain localization occurs at the rim of the holes in a thin metallic film and promotes crack formation in the film. Indeed, finite element analysis carried out using the COMSOL program for printed silver films with hole dimensions studied here and constrained to planar deformations (data shown in Supporting Information 1) indicate a 2- to 3 -fold increase in strain at the elongated hole's edge. The model further shows that if the film is able to flex out of plane, as when adhered to an elastomeric substrate, and if the hole edge-to-edge separation, $H$, is less than the hole diameter, $D$, then the network of holes acts to delocalize the strain by allowing serpentine-like stretching, thereby minimizing crack propagation in the film. However, it should be noted that the printed films studied have $3 D<H<10 D$ but nevertheless stretch.

Figure $4 a-c$ presents the morphological evolution of the printed silver conductors as a function of applied mechanical strain for the sample with $\lambda=12 \mu \mathrm{m}$. At $0 \%$ strain (before strain cycling), the printed film on PDMS is smooth and no cracks are visible. However, as the macroscopic uniaxial strain increases from 0 to $20 \%$, the conductor undergoes localized cracking in the direction normal to the stretch and compressive wrinkling in the stretch direction. During the first cycle (Figure 4a), transversal cracks form; their length matches the interpillar distance $\lambda$. Some of these cracks seem to start or stop at the closest pillar base at the site of local strain maxima as predicted by COMSOL simulations. At higher strains, the cracks widen but do not propagate, and additional narrower cracks appear in between the pillars. No longitudinal cracks form during the loading phase of cycle 1 .
After repeated cycling (Figure 4b), short longitudinal cracks are observed at $0 \%$ strain. The subsequent environmental SEM images recorded during stretching reveal limited aging of the printed silver film and almost identical morphology to images obtained during cycle 1 . Figure $4 \mathrm{c}$ shows the surface of a stretchable, 50-nm-thick evaporated gold film on smooth PDMS during the stretching cycle to $20 \%$ strain. The gold film on smooth PDMS has a built-in microtexture showing a random distribution of micrometer-long cracks. ${ }^{1,24}$ The cracks open during stretching and have remarkably similar morphologies to those observed on the printed silver films on patterned PDMS. Resistance data for the evaporated gold and printed silver films (shown in Supporting Information 2) are remarkably similar, both in form and evolution with repeated cycling. Note, however, that the printed line is 4 times thicker than the evaporated gold film and significantly thicker than the maximum stretchable thickness of evaporated gold of $\sim 100 \mathrm{~nm} .{ }^{26}$ Systematic image analysis carried out to determine the crack coverage area versus the sample surface area reveals that, after the initial cycle, the rate of change in the crack area is the same for an evaporated 50nm-thick gold film and a 200-nm-thick printed silver film (Figure 4d). However, the gold film has a larger initial crack density. ${ }^{24}$ Interestingly, it is possible to measure the strain in the printed film as a function of applied strain by measuring the average distance between pillars, along the stretching direction, on the series of ESEM images. Figure $4 \mathrm{e}$ plots the ratio between the measured strain and the applied strain as a function of the applied strain. During the first cycle, the interpillar strain in the printed film is lower than the macroscopic applied strain and then increases with applied strain. During the second cycle (ESEM 
images not shown), the interpillar strain and the applied strain match. This confirms that the formation of the microcracks in the printed film primarily occurs during the first cycle and evolves little upon further cycling. In Figure $4 a$ (cycle 1), the reduced stretching in between the pillars is compensated for by straining the pillars (in which the top sections become slightly ovoid). During the following cycles, the newly formed transversal cracks act as strain relief by taking up most of the elongation rather than the pillars and allowing the printed film to deflect locally out of plane. This stretchability mechanism is identical to that observed in a thin evaporated gold film on smooth PDMS. The presence of holes in the printed metallic film does not appear to affect this overall strain-relief mechanism.

\section{CONCLUSIONS}

In this study, we have demonstrated that (i) it is possible to print metallic inks onto an initially "hydrophobic" silicone substrate, (ii) micropillars arrays on PDMS guarantee wetting and pinning of the ink, and (iii) the printed conductors have a high conductivity $(11000 \mathrm{~S} / \mathrm{cm})$ and withstand large cyclic mechanical deformations. A generic method of forming a network of microcracks in an initially continuous and uniform conducting film of silicone is proposed and provides a technological route to producing highly elastic films of electronic device materials. The microfabricated and printed elastic conductors can be readily implemented in electronic and microsystem applications as stretchable interconnects for stretchable circuitry, compliant electrodes for transistors, supercapacitors or solar cells, and mechanically tunable antennas and rf components. Furthermore, we anticipate that this fabrication method may also be applied to a wide range of liquids and substrate patterns and thus has the potential for novel microfluidic applications.

\section{ASSOCIATED CONTENT}

S Supporting Information. COMSOL simulations of strained printed silver layers on pillar-patterned PDMS surfaces. Resistance data for evaporated Au films. This material is available free of charge via the Internet at http://pubs.acs.org.

\section{AUTHOR INFORMATION}

\section{Corresponding Author}

*E-mail: ar433@cam.ac.uk.

\section{ACKNOWLEDGMENT}

This work is supported by the EPSRC and a University Research Fellowship of the Royal Society to S.P.L.

\section{REFERENCES}

(1) Lacour, S. P.; Chan, D.; Wagner, S.; Li, T.; Suo, Z. Appl. Phys. Lett. 2006, 88, 204103.

(2) Brosteaux, D.; Axisa, F; Gonzalez, M; Vanfleteren, J. IEEE Electron Device Lett. 2007, 28, 552.

(3) Sekitani, T.; Noguchi, Y.; Hata, K.; Fukushima, T.; Aida, T.; Someya, T. Science 2008, 321, 1468.

(4) Sun, W.; Choi, M.; Jiang, H.; Huang, Y.; Rogers, J. A. Nat. Nanotechnol. 2007, 1, 201.

(5) Rosset, S.; Niklaus, M.; Dubois, P.; Shea, H. R. Adv. Funct. Mater. 2009, 19, 470.

(6) Cotton, D.; Graz, I. M.; Lacour, S. P. IEEE Sens. J. 2009, 9, 2008.
(7) Yu, Z.; Graudejus, O.; Tsay, C.; Lacour, S. P.; Wagner, S.; Morrison, B., III. J. Neurotrauma 2009, 26, 1135.

(8) Hu, L.; Pasta, M.; La Mantia, F.; Cui, L.; Jeong, S.; Deshazer, H. D.; Choi, J. W.; Han, S. M.; Cui, Y. Nano Lett. 2010, 10, 708.

(9) Gonzalez, M.; Axisa, F.; Vanden Bulcke, M.; Brosteaux, D.; Vandevelde, B.; Vanfleteren, J. Microelectron. Reliab. 2008, 48, 825.

(10) Gray, D. S.; Tien, J.; Chen, C. S. Adv. Mater. 2004, 16, 393.

(11) Park, J.; Lee, D. J.; Kim, S. J.; Oh, J. H. J. Micromech. Microeng. 2009, 19, 095021.

(12) InkTec.com.

(13) Ouyang, M.; Yuan, C.; Muisener, R. J.; Boulares, A.; Koberstein, J. T. Chem. Mater. 2000, 12, 1591.

(14) Im, M.; Im, H.; Lee, J.-H.; Yoon, J.-B.; Choi, Y.-K. Soft Matter 2010, 6, 1401.

(15) Yeo, J.; Choi, M. J.; Kim, D. S. J. Micromech. Microeng. 2010, 20, 025028 .

(16) Bico, J.; Tordeux, C.; Quéré, D. Europhys. Lett. 2001, 55, 214.

(17) Hay, K. M.; Dragila, M. I.; Liburdy, J. J. Colloid Interface Sci. 2008, 325, 472.

(18) Chau, T. T.; Bruckard, W. J.; Koh, P. T. L.; Nguyen, A. V. Adv. Colloid Interface Sci. 2009, 150, 106.

(19) Zografi, G.; Johnson, B. A. Int. J. Pharm. 1984, 22, 159.

(20) Harkema, S.; Schäffer, E.; Morariu, M. D.; Steiner, U. Langmuir 2003, 19, 9714-9718.

(21) Suh, K. Y.; Lee, H. H. Adv. Funct. Mater. 2002, 12, 405.

(22) Cubaud, T.; Jennner, P.; Fermigier, M. Proceedings of the Fourth European Coating Symposium, Advances in Coating Processes; Buchlin, J.-M., Anthoine, J., Eds.; Von Karman Institute: Brussels, Belgium, 2001; pp 73-78.

(23) Périchon-Lacour, S.; Wagner, S.; Huang, Z.; Suo, Z. Proc. Mater. Res. Soc. Fall Meet.; Materials Research Society: Warrendale, PA, 2002; Vol. 736, pp D4.8.1-D4.8.6.

(24) Graz, I. M.; Cotton, D. P. J.; Lacour, S. P. Appl. Phys. Lett. 2009, 94, 071902.

(25) Tucker, M. B.; Li, T. Int. J. Appl. Mech. 2009, 1, 557.

(26) Lacour, S. P.; Wagner, S.; Suo, Z. Mater. Res. Soc. Symp. Proc. 2004, 795, 193-198. 